\title{
Terrestrial slugs (Gastropoda, Pulmonata) in the NATURA 2000 areas of Cyprus island
}

\author{
Katerina Vardinoyannis', Simon Demetropoulos², Moissis Mylonas ${ }^{1,3}$, \\ Kostas A.Triantis ${ }^{4}$, Christodoulos Makris ${ }^{5}$, Gabriel Georgiou, \\ Andrzej Wiktor ${ }^{6}$, Andreas Demetropoulos ${ }^{7}$
}

I Natural History Museum of Crete, University of Crete, 71409 Herakleio Crete, Greece 2 Cyprus Wildlife Society, P.O.Box 24281, Lefkosia 1703, Cyprus 3 Department of Biology, University of Crete, 71409 Herakleio Crete, Greece 4 Natural History Museum of Crete, University of Crete, 71409 Herakleio Crete, Greece 521 Ethnikis Antistaseos, 3022 Limassol, Cyprus 6 Museum of Natural History, Wroctaw University, Sienkiewicza 21, 50-335 Wroctaw, Poland 7 Cyprus Wildlife Society, P.O.Box 24281, Lefkosia 1703, Cyprus

Corresponding author: Katerina Vardinoyannis (mollusca@nhmc.uoc.gr)

Academic editor: E. Neubert | Received 2 December 2011 | Accepted 22 February 2012 | Published 9 March 2012

Citation: Vardinoyannis K, Demetropoulos S, Mylonas M, Triantis KA, Makris C, Georgiou G, Wiktor A, Demetropoulos A (2012) Terrestrial slugs (Gastropoda, Pulmonata) in the NATURA 2000 areas of Cyprus island. ZooKeys 174: 63-77. doi: $10.3897 /$ zookeys. 174.2474

\begin{abstract}
Terrestrial slugs of the Island of Cyprus were recently studied in the framework of a study of the whole terrestrial malacofauna of the island. The present work was carried out in the Natura 2000 conservation areas of the island in 155 sampling sites over three years (2004-2007). Museum collections as well as literature references were included. In total six species are present in the Natura 2000 areas of the island, belonging to three families: Limacidae, Agriolimacidae and Milacidae. One of the species, Milax riedeli, is a new record for the island. The distribution of the species across the island and in the surrounding areas is discussed.
\end{abstract}

\section{Keywords}

Agriolimacidae, Limacidae, Milacidae, Distribution, East Mediterranean

\section{Introduction}

Cyprus, the largest island in the Eastern Mediterranean, has an area of 9,251 $\mathrm{km}^{2}$, and there are 33 special areas under nature conservation, that cover $22 \%$ of the total area. The designation of these areas was based mainly on habitat types, geology, knowledge

Copyright Katerina Vardinoyannis et al. This is an open access article distributed under the terms of the Creative Commons Attribution License 3.0 (CC-BY), which permits unrestricted use, distribution, and reproduction in any medium, provided the original author and source are credited. 
of plant and vertebrate species, and on published data on invertebrates. This is in accordance with what Dimitrakopoulos et al. (2004) claim "... it is questionable whether the inclusion of species and habitats in the list of biodiversity components of 'community interest' has been based on a previous detailed evaluation of regional biodiversity patterns, but rather the selection was based on the inclusion of pre-existing national ad hoc schemes".

Our previous knowledge of the slugs of Cyprus was mainly based on a fairly recent paper by Rähle (1991). In most other relevant papers slugs of Cyprus appear in passing, either because the work deals mainly with the neighbouring areas of the Mediterranean (Schütt 2005, Heller 2009) or they concern a particular slug taxon (Hesse 1926). Six slug species had been recorded from the island, but only three of them within a NATURA 2000 conservation area; namely Deroceras berytensis, D. chrysorroyatissensis and Limax flavus (Rähle 1984, 1991; Wiktor 2001). These slugs are recorded from six conservation areas, which are among the most popular and most visited parts of the island.

In this work we present new distributional data about the slugs of Cyprus and comments on their taxonomy when necessary. Additionally, we discuss their presence in the conservation areas and the whole island and compare it with occurrence in surrounding countries.

\section{Material and methods}

During the years 2004-2007 we collected land snails in all NATURA 2000 areas. Slugs were found at 99 sites within 28 of the 33 areas although we collected land snails at 155 sampling sites (Map 1, Table 1). Sampling sites were intended to cover the whole diversity (habitat and substrate) of each NATURA 2000 area. Snails and slugs were collected only during the wet period (October-April) by A. Demetropoulos (AD), S. Demetropoulos (SD), Chr. Makris (M), Chr. Makris \& L. Georgiou (MG), M. Mylonas (MM), K.A. Triantis, and K. Vardinoyannis (V). We also included material from the mollusc collection of the Natural History Museum of Crete (Map 1, Table 1). After sampling, specimens were relaxed and then preserved in $75 \%$ ethanol. Their identification was based on anatomy of the genitalia. The material is kept in the Natural History Museum of Crete and in the Museum of Natural History, Wrocław University.

Table I. Sampling sites in each NATURA 2000 area, the date of collection, the vegetation type and the dominant plant species, the substrate and the corresponding number on Map 1.

\begin{tabular}{|c|c|c|c|c|c|}
\hline NATURA 2000 area & Sampling site with slugs & Date & $\begin{array}{l}\text { Number } \\
\text { on map }\end{array}$ & Dominant Vegetation & Substrate \\
\hline \multirow[b]{2}{*}{ Agiatis } & gia S (CY411-1) & 006 & 25 & \multirow[b]{2}{*}{ Pine forest (Pinus brutia) } & \multirow[b]{2}{*}{$\begin{array}{l}\text { Diabase } \\
\text { dykes }\end{array}$} \\
\hline & $\begin{array}{l}\text { Agiatis-500m from Tarmac } \\
\text { CY411-2 }\end{array}$ & $14 / 3 / 2006$ & 26 & & \\
\hline $\begin{array}{l}\text { Akrotirio Aspro-Pe- } \\
\text { tra tou Romiou }\end{array}$ & $\begin{array}{l}\text { Aspro cape-Petra tou Ro- } \\
\text { miou (eastern valley) }\end{array}$ & $29 / 1 / 2005$ & 53 & $\begin{array}{l}\text { Phrygana (Sarcopoterium } \\
\text { spinosum; Maquis (Olea } \\
\text { europaea, Ceratonia } \\
\text { siliqua) }\end{array}$ & $\begin{array}{l}\text { Biocalca- } \\
\text { renites, } \\
\text { sandstones }\end{array}$ \\
\hline
\end{tabular}




\begin{tabular}{|c|c|c|c|c|c|}
\hline NATURA 2000 area & Sampling site with slugs & Date & $\begin{array}{l}\text { Number } \\
\text { on map }\end{array}$ & Dominant Vegetation & Substrate \\
\hline \multirow[b]{2}{*}{ Alykes Larnakas } & Alyki, Tekes (site 1) & $15 / 1 / 2005$ & 95 & \multirow[b]{2}{*}{ Plantations with Acacia } & \multirow[b]{2}{*}{ Sand, silts } \\
\hline & $\begin{array}{l}\text { Larnaka salt marsh new } \\
\text { buildings }\end{array}$ & $19 / 11 / 2005$ & 96 & & \\
\hline $\begin{array}{l}\text { Alykos Potamos-Ag- } \\
\text { ios Sozomenos }\end{array}$ & Kotsiatis Dam (CY202-1) & $4 / 3 / 2006$ & 81 & Phrygana (S. spinosum) & $\begin{array}{l}\text { Pillow la- } \\
\text { vas, Olivine } \\
\text { - Pyroxene }\end{array}$ \\
\hline \multirow[t]{2}{*}{ Cha Potami } & $\begin{array}{l}\text { Cha river, Kato Archi- } \\
\text { mantria }\end{array}$ & $5 / 3 / 2005$ & 52 & \multirow{2}{*}{$\begin{array}{l}\text { Maquis (O. europaea } \& \\
\text { C. siliqua) }\end{array}$} & \multirow{2}{*}{$\begin{array}{l}\text { Chalks, } \\
\text { marls }\end{array}$} \\
\hline & Cha river, Orites & $5 / 3 / 2005$ & 51 & & \\
\hline \multirow{10}{*}{ Chersonisos Akama } & Agios Kononas springs & $24 / 11 / 2005$ & 1 & \multirow{10}{*}{$\begin{array}{l}\text { Maquis (Juniperus } \\
\text { phoenicea, O. europaea, } \\
\text { C. siliqua); } \\
\text { Phrygana (S. spinosum); } \\
\text { Pine forests (P. brutia) }\end{array}$} & \multirow{10}{*}{ Limestone } \\
\hline & $\begin{array}{l}\text { Agios Minas spring } \\
\text { CY410-11 }\end{array}$ & $15 / 3 / 2005$ & 4 & & \\
\hline & $\begin{array}{l}\text { Akamas (may be Loutra tis } \\
\text { Afroditis) }\end{array}$ & $1 / 10 / 1989$ & 3 & & \\
\hline & Akamas forest CY 410-8 & $12 / 3 / 2005$ & 6 & & \\
\hline & $\begin{array}{l}\text { Akamas, Loutra tis Afrodi- } \\
\text { tis CY 410-10 }\end{array}$ & $15 / 3 / 2005$ & 2 & & \\
\hline & Avakas & $19 / 2 / 2005$ & 8 & & \\
\hline & $\begin{array}{l}\text { Mountiko maquis } \\
\text { CY410-3 }\end{array}$ & $24 / 1 / 2005$ & 9 & & \\
\hline & Petratis gorge & $23 / 11 / 2005$ & 7 & & \\
\hline & Pittokopos CY410-13 & $16 / 3 / 2005$ & 5 & & \\
\hline & Pykni forest CY410-4 & $15 / 2 / 2005$ & 10 & & \\
\hline \multirow{4}{*}{$\begin{array}{l}\text { Dasos Lemesou- } \\
\text { Periochi Kyparisia }\end{array}$} & $\begin{array}{l}\text { Germasogeia north, at the } \\
\text { stream (CY 20) }\end{array}$ & $20 / 2 / 2000$ & 59 & \multirow{4}{*}{$\begin{array}{l}\text { Pine forest (P. brutia); } \\
\text { Maquis }(O \text {. europaea, } \\
\text { C. siliqua) }\end{array}$} & \multirow{4}{*}{$\begin{array}{l}\text { Serpen- } \\
\text { tinized } \\
\text { harzburgi- } \\
\text { tes, Diabase } \\
\text { dykes and } \\
\text { gabbros }\end{array}$} \\
\hline & $\begin{array}{l}\text { Lemesos forest, Akrounta } \\
\text { river valley }\end{array}$ & $11 / 2 / 2006$ & 62 & & \\
\hline & Lemesos forest, Foinikaria & $2 / 1 / 2005$ & 61 & & \\
\hline & $\begin{array}{l}\text { Lemesos forest, Germaso- } \\
\text { geia dam, Foinikaria }\end{array}$ & $2 / 1 / 2005$ & 60 & & \\
\hline \multirow{7}{*}{ Dasos Machaira } & Gionia Camp (CY204-2) & $17 / 3 / 2006$ & 87 & \multirow{7}{*}{$\begin{array}{l}\text { Forest }(Q \text {. alnifolia, } P \text {. } \\
\text { brutia) }\end{array}$} & \multirow{7}{*}{$\begin{array}{l}\text { Diabase } \\
\text { dykes and } \\
\text { pillow lavas }\end{array}$} \\
\hline & $\begin{array}{l}\text { Gionia Valley north } \\
\text { (CY204-3) }\end{array}$ & $17 / 3 / 2006$ & 85 & & \\
\hline & Kapedes (CY204-1) & $17 / 3 / 2006$ & 82 & & \\
\hline & Kiona $-1380 \mathrm{~m}$ alt & $22 / 12 / 2006$ & 86 & & \\
\hline & Lazania CY 204-7 & $27 / 12 / 2006$ & 84 & & \\
\hline & $\begin{array}{l}\text { Machairas, Kyprovasa- Ar- } \\
\text { katzi tou Klosmatou }\end{array}$ & $22 / 12 / 2006$ & 88 & & \\
\hline & Philani Pine CY 204-6 & $27 / 12 / 2006$ & 83 & & \\
\hline \multirow{7}{*}{ Dasos Pafou } & Dasos Pafou Tripyla & $7 / 1 / 2006$ & 29 & \multirow{7}{*}{$\begin{array}{l}\text { Forests with Pines ( } P \\
\text { brutia) and Cedars } \\
\text { (Cedrus brevifolia) }\end{array}$} & \multirow{7}{*}{$\begin{array}{l}\text { Diabase } \\
\text { dykes }\end{array}$} \\
\hline & Gerakies (CY 206-3) & $15 / 3 / 2006$ & 32 & & \\
\hline & Kedron valley & $13 / 3 / 2005$ & 28 & & \\
\hline & Kedron valley (CY 10) & $18 / 2 / 2000$ & 27 & & \\
\hline & $\begin{array}{l}\text { Kremnos tis Pellis CY } \\
206-1\end{array}$ & $15 / 3 / 2006$ & 30 & & \\
\hline & Roudia Bridge (site CY7) & $17 / 2 / 2000$ & 33 & & \\
\hline & $\begin{array}{l}\text { Sylladin tou Petrou near } \\
\text { Tsakistra }\end{array}$ & $15 / 3 / 2006$ & 31 & & \\
\hline
\end{tabular}




\begin{tabular}{|c|c|c|c|c|c|}
\hline NATURA 2000 area & Sampling site with slugs & Date & $\begin{array}{l}\text { Number } \\
\text { on map }\end{array}$ & Dominant Vegetation & Substrate \\
\hline \multirow{3}{*}{ Dasos Stavrovouniou } & $\begin{array}{l}\text { Stavrovouni, entrance of } \\
\text { monastery }\end{array}$ & $19 / 12 / 2006$ & 93 & \multirow{3}{*}{$\begin{array}{l}\text { Pine forest (P. brutia); } \\
\text { Maquis }(O \text {. europaea, } \\
\text { C. siliqua) }\end{array}$} & \multirow{3}{*}{$\begin{array}{l}\text { Diabase } \\
\text { dykes and } \\
\text { pillow lavas }\end{array}$} \\
\hline & Stavrovouni, low & $19 / 12 / 2006$ & 92 & & \\
\hline & Stavrovouni, NE low & $19 / 12 / 2006$ & 94 & & \\
\hline \multirow[b]{2}{*}{ Drymou } & Drymou Oak & $28 / 3 / 2007$ & 16 & \multirow[b]{2}{*}{ Riparian } & \multirow{2}{*}{$\begin{array}{l}\text { Biocalca- } \\
\text { renites, } \\
\text { sandstones } \\
\end{array}$} \\
\hline & Drymou Valley & $28 / 3 / 2007$ & 15 & & \\
\hline \multirow{2}{*}{$\begin{array}{l}\text { Episkopi tou Morou } \\
\text { Nerou }\end{array}$} & Ezousas Alder (CY405-1) & $11 / 2 / 2006$ & 22 & \multirow[b]{2}{*}{ Maquis (Q. coccifera) } & \multirow{2}{*}{$\begin{array}{l}\text { Sand, silts, } \\
\text { clays }\end{array}$} \\
\hline & $\begin{array}{l}\text { Ezousas Pseudogarrigue } \\
(\mathrm{CY} 405-2)\end{array}$ & $11 / 2 / 2006$ & 21 & & \\
\hline \multirow{4}{*}{$\begin{array}{l}\text { Ethniko Dasiko } \\
\text { Parko Troodous }\end{array}$} & Caledonian falls & $22 / 11 / 2005$ & 63 & \multirow{4}{*}{$\begin{array}{l}\text { Pine forests (P.nigra, } P \text {. } \\
\text { brutia) }\end{array}$} & \multirow{4}{*}{$\begin{array}{l}\text { Gabbros, } \\
\text { harzbur- } \\
\text { gites }\end{array}$} \\
\hline & Mesapotamos waterfall & $22 / 11 / 2005$ & 65 & & \\
\hline & Platres to Mesapotamos & $22 / 11 / 2005$ & 64 & & \\
\hline & Troodos mt., Almyrolivado & $26 / 11 / 2005$ & 66 & & \\
\hline \multirow{3}{*}{ Kavo Gkreko } & Cavo Gkreko Rocks & $9 / 1 / 2005$ & 99 & \multirow{3}{*}{$\begin{array}{l}\text { Maquis }(J \cdot \text { phoenicea }) \\
\text { Phrygana }(S . \text { spinosum })\end{array}$} & \multirow{3}{*}{ Limestone } \\
\hline & $\begin{array}{l}\text { Gkreco cape at Agioi Anar- } \\
\text { gyroi (CY 24) }\end{array}$ & $21 / 2 / 2000$ & 98 & & \\
\hline & Paralimniou lake & $9 / 1 / 2005$ & 97 & & \\
\hline \multirow{6}{*}{ Koilada Diarizou } & Diarizos Arminou & $28 / 1 / 2006$ & 49 & \multirow{6}{*}{$\begin{array}{l}\text { Maquis (O. europaea, } C \text {. } \\
\text { siliqua); } \\
\text { Phrygana }(S . \text { spinosum); } \\
\text { Pine forests }(P \text {. brutia) }\end{array}$} & \multirow{6}{*}{$\begin{array}{l}\text { Chalks, } \\
\text { sand, lava } \\
\text { breccia }\end{array}$} \\
\hline & $\begin{array}{l}\begin{array}{l}\text { Diarizos gorge, after Kikisia } \\
(\mathrm{CY} 8)\end{array} \\
\end{array}$ & $17 / 2 / 2000$ & 47 & & \\
\hline & Diarizos Kidasi & $22 / 1 / 2006$ & 45 & & \\
\hline & Diarizos Nikokleia & $28 / 1 / 2006$ & 44 & & \\
\hline & Diarizos valley, Gefyri & $22 / 1 / 2005$ & 48 & & \\
\hline & $\begin{array}{l}\text { Diarizos valley, Petres ton } \\
\text { Hasanpoulion }\end{array}$ & $22 / 1 / 2005$ & 46 & & \\
\hline \multirow{5}{*}{ Koilada Limnati } & $\begin{array}{l}\text { Limnatis valley, } 1 \mathrm{~km} \text { west } \\
\text { of the bridge, } 400 \mathrm{~m} \text { alt. }\end{array}$ & $5 / 12 / 2004$ & 58 & \multirow{5}{*}{$\begin{array}{l}\text { Maquis (O. europaea, } C . \\
\text { siliqua) }\end{array}$} & \multirow{5}{*}{$\begin{array}{l}\text { Chalks, ser- } \\
\text { pentinite }\end{array}$} \\
\hline & Limnatis valley, Alassa & $19 / 11 / 2005$ & 55 & & \\
\hline & Limnatis valley, Mantra & $18 / 2 / 2006$ & 54 & & \\
\hline & $\begin{array}{l}\text { Palia Korfi, approx. } 500 \mathrm{~m} \\
\text { alt., Limnatis valley }\end{array}$ & $4 / 12 / 2004$ & 56 & & \\
\hline & $\begin{array}{l}\text { Palia Korf, river below at } \\
\text { Limnatis bridge }\end{array}$ & $4 / 12 / 2004$ & 57 & & \\
\hline \multirow[t]{2}{*}{ Kritou Marotou } & $\begin{array}{l}\text { Kritou Marotou Cultiva- } \\
\text { tions }\end{array}$ & $27 / 3 / 2007$ & 24 & \multirow{2}{*}{$\begin{array}{l}\text { Riparian and cultiva- } \\
\text { tions }\end{array}$} & \multirow{2}{*}{$\begin{array}{l}\text { Chalks, } \\
\text { marls, clays }\end{array}$} \\
\hline & Kritou Marotou Oak & $27 / 3 / 2007$ & 23 & & \\
\hline \multirow{3}{*}{ Lefkaron } & Lefkara-Agios Minas & $25 / 2 / 2006$ & 91 & \multirow{3}{*}{$\begin{array}{l}\text { Presteppe scrub (Genista } \\
\text { fasselata; Maquis }(Q . \\
\text { coccifera) }\end{array}$} & \multirow{3}{*}{$\begin{array}{l}\text { Chalks, } \\
\text { marls and } \\
\text { pillow lavas }\end{array}$} \\
\hline & Lefkara $600 \mathrm{~m}$ alt & $19 / 11 / 2005$ & 89 & & \\
\hline & $\begin{array}{l}\text { Lefkara croosroad to Kato } \\
\text { Drys }\end{array}$ & $11 / 3 / 2006$ & 90 & & \\
\hline & Kyperounda (CY205-1) & $25 / 3 / 2006$ & 67 & & \\
\hline & Lagoudera valley & $20 / 12 / 2006$ & 70 & & \\
\hline & Lagoudera, $6 \mathrm{~km}$ north & $21 / 12 / 2006$ & 73 & & \\
\hline & Papoutsa $\sim 1240 \mathrm{~m}$ alt. & $20 / 12 / 2006$ & 72 & Forest (Q. alnifolia, $P$. & Diabase \\
\hline Madari-Papoutsa & Pitsilia district, Kyperounta & $15 / 4 / 2001$ & 68 & brutia) & dykes and \\
\hline & Polystypos Fountoukies & $17 / 12 / 2006$ & 71 & & \\
\hline & Spilia-Madari, $1250 \mathrm{~m}$ alt. & $17 / 12 / 2006$ & 69 & & \\
\hline & Xyliati dam, low & $21 / 12 / 2006$ & 74 & & \\
\hline
\end{tabular}




\begin{tabular}{|c|c|c|c|c|c|}
\hline NATURA 2000 area & Sampling site with slugs & Date & $\begin{array}{l}\text { Number } \\
\text { on map }\end{array}$ & Dominant Vegetation & Substrate \\
\hline Mammari-Deneia & Mammari 1st site & $21 / 11 / 2005$ & 80 & Phrygana (S. spinosum) & $\begin{array}{l}\text { Biocalca- } \\
\text { renites, } \\
\text { sandstones } \\
\end{array}$ \\
\hline \multirow{3}{*}{ Maroullena } & Maroulena gorge & $17 / 12 / 2006$ & 75 & \multirow{3}{*}{$\begin{array}{l}\text { Pine forest (P. brutia) } \\
\text { and Riparian }\end{array}$} & \multirow{3}{*}{ Pillow lavas } \\
\hline & Maroulena's Dam & $23 / 12 / 2006$ & 76 & & \\
\hline & Maroulena's Pine & $23 / 12 / 2006$ & 77 & & \\
\hline \multirow{4}{*}{ Mavrokolympos } & $\begin{array}{l}\text { Agios Neophytos Valley } \\
\text { CY408-4 }\end{array}$ & $13 / 1 / 2006$ & 20 & \multirow{4}{*}{$\begin{array}{l}\text { Maquis (O. europaea, } \\
\text { C. siliqua); Phrygana }(S . \\
\text { spinosum) }\end{array}$} & \multirow{4}{*}{$\begin{array}{l}\text { Chalks, } \\
\text { marls }\end{array}$} \\
\hline & $\begin{array}{l}\text { Agios Neophytos Garrigue } \\
\text { CY408-3 }\end{array}$ & $13 / 1 / 2006$ & 19 & & \\
\hline & $\begin{array}{l}\text { Mavrokolymbos Garrigue } \\
\text { CY408-1 }\end{array}$ & $9 / 1 / 2006$ & 18 & & \\
\hline & $\begin{array}{l}\text { Mavrokolymbos Stream } \\
\text { CY408-2 }\end{array}$ & $9 / 1 / 2006$ & 17 & & \\
\hline \multirow[b]{2}{*}{ Periochi Mitserou } & $\begin{array}{l}\text { Mitsero-Agios Panteleimo- } \\
\text { nas }\end{array}$ & $11 / 3 / 2006$ & 79 & \multirow{2}{*}{$\begin{array}{l}\text { Phrygana (S. spinosum); } \\
\text { Pine forest (P. brutia) }\end{array}$} & \multirow{2}{*}{$\begin{array}{l}\text { Chalk \& } \\
\text { Limestone }\end{array}$} \\
\hline & Mitsero Pinewood \& valley & $11 / 3 / 2006$ & 78 & & \\
\hline Platy & $\begin{array}{l}\text { Platy area, crossroad Kele- } \\
\text { fos-Kaminaria-Milikouri }\end{array}$ & $19 / 2 / 2005$ & 50 & Pine forest (P. brutia) & $\begin{array}{l}\text { Diabase } \\
\text { dykes }\end{array}$ \\
\hline Polis Gialia & Gialia Acacia CY401-1 & $8 / 1 / 2006$ & 14 & Plantations with Acacia & $\begin{array}{l}\text { Calcaren- } \\
\text { ites, sands, } \\
\text { gravel }\end{array}$ \\
\hline \multirow{3}{*}{ Skoulli } & $\begin{array}{l}\text { Chrysochou River } \\
\text { CY409-2 }\end{array}$ & $15 / 1 / 2006$ & 12 & \multirow{3}{*}{$\begin{array}{l}\text { Woodland (Q.infectoria); } \\
\text { Riparian (Platanus orien- } \\
\text { talis, Nerium oleander) }\end{array}$} & \multirow{3}{*}{$\begin{array}{l}\text { Sand, silts, } \\
\text { gravel }\end{array}$} \\
\hline & Goudi Oak CY409-1 & $15 / 1 / 2006$ & 11 & & \\
\hline & Polis Camp river CY409-3 & $15 / 1 / 2006$ & 13 & & \\
\hline \multirow[t]{4}{*}{ Vouni Panagias } & Agia Moni south of Panagia & $22 / 12 / 2004$ & 34 & $\begin{array}{l}\text { Forests (Q. infectoria, } \\
\text { Pinus brutia) }\end{array}$ & $\begin{array}{l}\text { Chalks, } \\
\text { marls }\end{array}$ \\
\hline & $\begin{array}{l}\text { Makries Limnes Chasan- } \\
\text { poulion-Eryfiou-Profitis } \\
\text { Ilias }\end{array}$ & $23 / 12 / 2004$ & 36 & & \\
\hline & \begin{tabular}{|l|} 
Profitis Ilias \\
\end{tabular} & $1 / 2 / 2005$ & 35 & & \\
\hline & Vloudkia & $23 / 12 / 2004$ & 37 & & \\
\hline \multirow{6}{*}{ Xeros Potamos } & $\begin{array}{l}\text { Asprokremnos Pools } \\
\text { CY407-1 }\end{array}$ & $2 / 2 / 2006$ & 43 & \multirow{6}{*}{$\begin{array}{l}\text { Phrygana (S. spinosum); } \\
\text { Maquis (O. europaea, C. } \\
\text { siliqua); } \\
\text { Pine forests ( } P \text {. brutia) }\end{array}$} & \multirow{6}{*}{$\begin{array}{l}\text { Chalks, } \\
\text { marls }\end{array}$} \\
\hline & \begin{tabular}{|l|} 
Finikas CY407-2 \\
\end{tabular} & $2 / 2 / 2006$ & 42 & & \\
\hline & Nata Pine CY407-5 & $6 / 2 / 2006$ & 39 & & \\
\hline & Xeros Army CY407-3 & $2 / 2 / 2006$ & 41 & & \\
\hline & Xeros Rock CY407-4a & $3 / 2 / 2006$ & 38 & & \\
\hline & Xirou valley, Nata & $5 / 2 / 2005$ & 40 & & \\
\hline \multirow{2}{*}{ Stavros tis Psokas } & Agios Merkourios & & & \multirow{2}{*}{ Pine forests } & \multirow{2}{*}{$\begin{array}{l}\text { Diabase } \\
\text { dykes }\end{array}$} \\
\hline & Stavros tis Psokas (CY 11) & $18 / 2 / 2000$ & & & \\
\hline \multicolumn{6}{|l|}{$\begin{array}{l}\text { Ethniko Dasiko } \\
\text { Parko Rizoelias }\end{array}$} \\
\hline Asgata & \multirow{4}{*}{$\begin{array}{l}\text { No slugs were found } \\
\text { in these areas }\end{array}$} & & & & \\
\hline Lympion-Agia Anna & & & & & \\
\hline Peristerona & & & & & \\
\hline Koilada Kargotis & & & & & \\
\hline
\end{tabular}

In Table 1 we give the sampling sites in each NATURA 2000 area, the date of collection, the vegetation type, the dominant plant and the substrate.

Initially we assembled all data from the literature, including the doubtful names. These are presented and discussed separately for each species. 


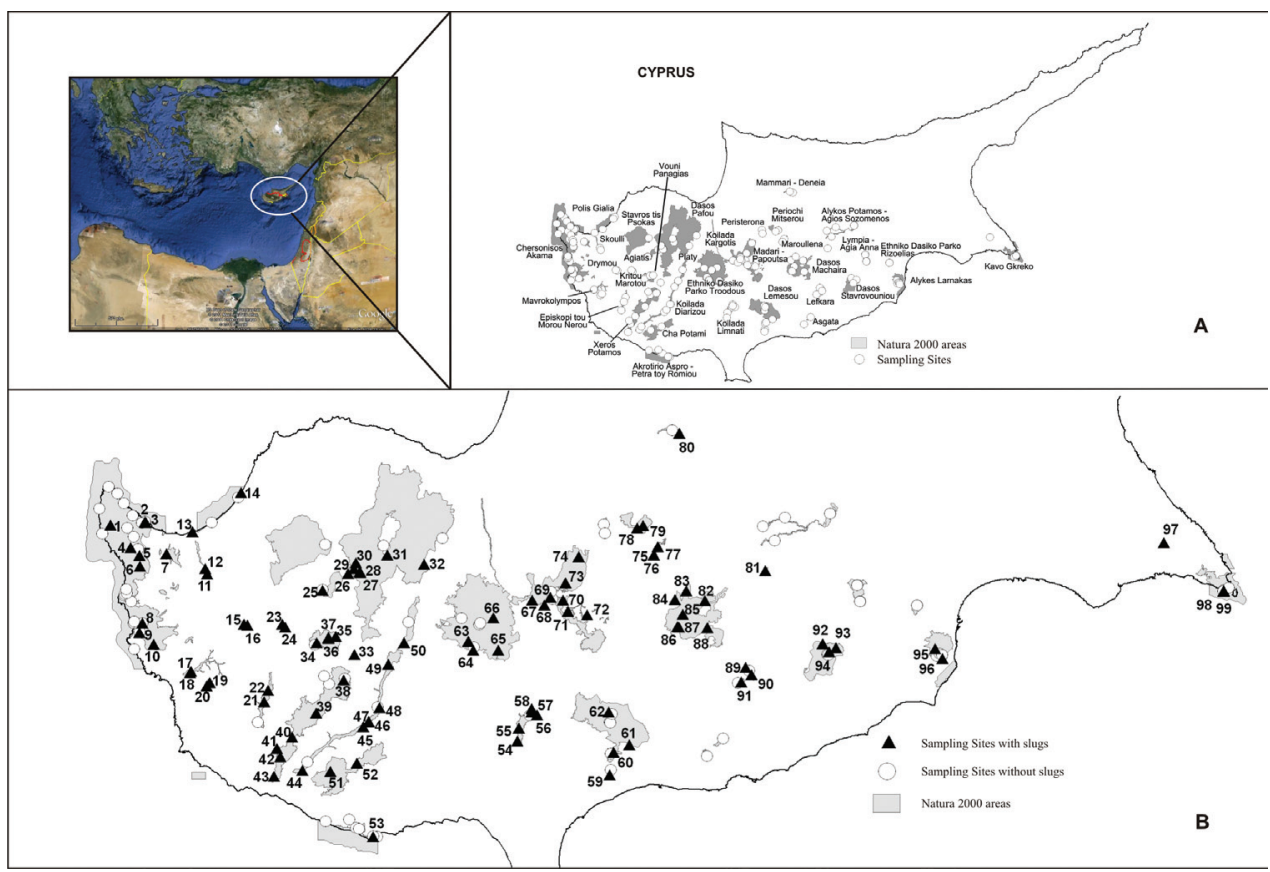

Map I. Natura 2000 areas A and sampling sites B in each area. Numbers depict sampling sites where slugs were found (their name is given in Table 1)

\section{Results}

In total we found six slug species belonging to three families - Agriolimacidae, Limacidae and Milacidae. Below we present analytically for each species the collecting sites in each NATURA 2000 conservation area (bold), data from literature, and if necessary comments on its systematics.

\section{Family Agriolimacidae Wagner, 1935}

\section{Deroceras berytensis (Bourguignat, 1852)}

This species had been reported from Akrotirio Aspro-Petra tou Romiou; Alykes Larnakas; Chersonisos Akama; Ethniko Dasiko Parko Troodous; Koilada Diarizou; Polis Gialia and Vouni Panagias (Rähle 1984, 1991).

We found it in (Fig. 1a): Alykes Larnakas: Larnaka salt marsh new buldings, 19.11.05, M; Alyki Tekes, 15.1.05, MG. Alykos Potamos - Agios Sozomenos: Kotsiatis Dam (CY202-1), 4.3.2006, SD. Cha Potami: Cha river, Kato Archimandria, 5.03.05, MG. Chersonisos Akama: Agios Minas spring CY 410-11, 15.3.05, AD; Agios Kononas springs, 24/11/2005, AD; Akamas (may be Loutra tis Afroditis), 1.10.1989, MM; Petratis gorge, 23.11.05, MM; Pittokopos CY 410-13, 16.03.05, 

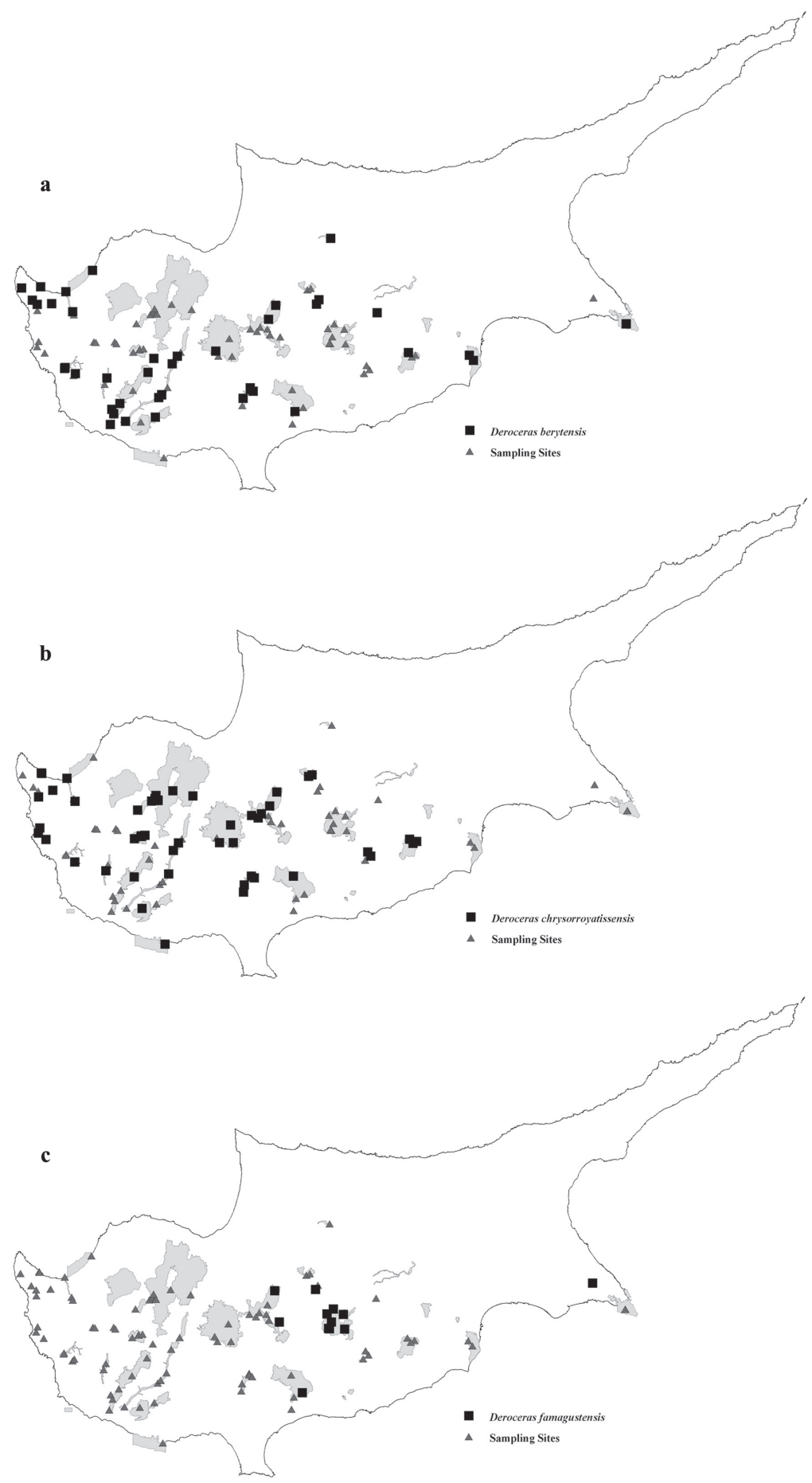

Figure I. Distribution of a D. berytensis $\mathbf{b}$ D. chrysorroyatissensis and $\mathbf{c} D$. famagustensis in the Natura 2000 areas of conservation in Cyprus Island. 
SD. Dasos Lemesou - Periochi Kyparisia: Lemesos forest, Germasogeia dam, Foinikaria, 2.1.05 MG. Dasos Pafou: Roudia bridge (CY7), 17.2.00, MM. Dasos Stavrovouniou: Stavrovouni, low, 19.12.06 MM. Episkopi tou Morou Nerou: Ezousas Alder (CY405-1), 11.02.06, SD. Kavo Gkreko: Cavo Greko Rocks, 9.1.05, MM; Gkreko cape at Agioi Anagyroi (CY24), 21.02.00, MM. Koilada Diarizou: Diarizou gorge, after Kikisia (CY 8), 17.02.00, MM; Diarizos Arminou, 28.1.06, MG; Diarisos Kidasi, 22.1.06, AD; Diarizos Nikokleia, 28.1.06, MG; Diarizos valley, Petres ton Hasanpoulion, 22.1.2005, MG. Koilada Limnati: Limnatis valley, Alassa, 19.11.05, MG; Palia Korfi, river below at Limnatis bridge, 4.12.04, MM; Palia Korfi, approx. 500 $\mathrm{m}$ alt. Limnatis valley, 4.12.04, MM; Limnatis valley, $1 \mathrm{~km}$ west of the bridge, $400 \mathrm{~m}$ alt., 4/12/2004, MM. Madari - Papoutsa: Lagoudera, 6 km north, 21.12.06, V; Xyliati dam, low, 21.12.06, MM. Mammari - Deneia: Mammari 1st site, 21.11.2005, V. Maroullena: Maroulena's Dam, 23.12.06, SD; Maroulena gorge, 17.12.06, MM, V, SD. Mavrokolympos: Mavrokolymbos stream CY408-2, 9.1.06, SD; Mavrokolymbos Garrigue CY408-1, 9.01.06 SD; Agios Neophytos valley (CY 408-4), 13.1 .06 SD. Polis Gialia: Gialia Acacia CY401-1, 8.01.06, AD \& SD. Skoulli: Chrysochou River CY 409-2, 15.01.06, SD; Polis Camp river CY409-3, 15.01.06, SD. Xeros Potamos: Asprokremnos Pools CY407-1, 2.2.06, AD; Xirou valley Nata, 5.2.05 MG; Finikas CY407-2, 2.02.06, AD \& SD; Xeros Army (CY 407-3), 2.2.06, AD \& SD; Xeros Rock CY407-4a, 3.02.06, AD \& SD

\section{Comments on systematic characters}

The colour of alcohol-preserved specimens ranges from cream to dark brown, especially on the back and mantle; the dark pigment is distributed more or less evenly with no distinct spots; when spots are present, they are blurred and their margins fuse (they are never black dots). These external characters are always combined with the following anatomical characters: long caecum, forked penial gland with a distinct common stalk, more or less half as long as the entire gland. The two branches of the penial gland are covered by glandular papillae. The stimulator in the penis is wide, flat, and bowl-shaped.

The species varies very widely (Wiktor 2000); a few very similar slugs, of unclear taxonomic status, are found in the literature. Agriolimax cyprius Simroth, 1906 was described from Cyprus as two forms (A. cyprius and A. cyprius coeciger Simroth, 1906). The description, however, is so laconic that it is impossible to say which slugs Simroth (1906) was dealing with (the types most probably have not been preserved). Rähle (1984) commented on these slugs saying that the slugs from Cyprus differed in a few anatomical details from $D$. berytensis earlier described from Lebanon. The slugs from Cyprus are often smaller; differ somewhat in the appearance of their glandula hermaphroditica, caecum length, penial gland and stimulator. Rähle (1984) suspected that it might be only a form of the variable $D$. berytensis. In our opinion these comments 
are justified and agree with our own observations; according to Wiktor (2000), at the current state of knowledge it is reasonable to regard the name $A$. cyprium as a junior synonym of the widely distributed and very variable $D$. berytensis, rather than use names without knowing what they refer to. It cannot be excluded that Simroth (1906) was dealing not only with $D$. berytensis but also for example with $D$. famagustensis.

\section{Deroceras chrysorroyatissensis Rähle, 1984}

This species had been reported from Akrotirio Aspro-Petra tou Romiou and Vouni Panagias (Rähle 1991).

We found it in (Fig. 1b): Agiatis: Agiatis-Agia S (CY411-1), 14.03.06, MM, V, AD, SD; Agiatis - 500m from Tarmac CY411-2, 14.03.06, MM, V, AD, SD. Akrotirio Aspro - Petra tou Romiou: Aspro cape - Petra tou Romiou (eastern valley), 29.01.05, M. Cha Potami: Cha river Orites, 5.03.05, MG. Chersonisos Akama: Pykni forest CY 410-4, 15.02.05, SD ? juv.; Akamas (may be Loutra tis Afroditis), 1.10.89, MM; Akamas, Loutra tis Afroditis CY410-10, 15.03.05, SD; Avakas, 19.02.05, SD; Mountiko maquis CY410-3, 24.01.05, SD ? juv.; Petratis gorge, 23.11.05, MM; Akamas forest CY 410-8, 12.03.05, SD. Dasos Lemesou - Periochi Kyparisia: Lemesos forest, Akrounta river valley, 2.01.06, MG. Dasos Pafou: Gerakies CY206-3, 15.03.06, MM, V, AD, SD; Kremnos tis Pellis CY 206-1, 15.3.06. MM, V, AD, SD; Sylladin tou Petrou CY206-2, 15.03.06, MM, V, AD, SD; Kedron valley, 13.03.05, MG; Dasos Pafou-Tripyla, 7.01.06, AD; Kedron valley (CY 10), 18.02.00, V. Dasos Stavrovouniou: Stavrovouni, entrance of monastery, 19.12.06, MM, V; Stavrovouni, NE low, 19.12.06, MM, V; Stavrovouni, low, 19.12.06, MM, V. Episkopi tou Morou Nerou: Ezousas Pseudogarrigue (CY 405-2), 11.02.06, SD. Ethniko Dasiko Parko Troodous: Mesapotamos waterfall, 22.11.05, V; Troodos mt. Almyrolivado, 26.11.05, M; Platres to Mesapotamos, 22.11.05, MM. Koilada Diarizou: Diarizos Arminou, 28.01.06, MG; Diarizou valley, Gefyri, 22.1.05, MG. Koilada Limnati: Limnatis valley, Alassa, 19.11.05, MG; Palia Korfi, river below at Limnatis bridge, 5.12.04, MG; Palia Korfi, approx. 500m alt. Limnatis valley, 5.12.04, MM; Limnatis valley, Mantra, 18.02.06, M. Lefkaron: Lefkara 600m alt., 19.11.05, MM. ? juv.; Lefkara croosroad to Kato Drys, 11.03.06, MM. Madari - Papoutsa: Xyliati dam, low, 21.12.06. MM, V; Lagoudera 6 km north, 21.12.06, MM, V; Pitsilia district, Kyperounta, 15.04.01 ? juv.; Kyperounta (CY 205-1), 25.03.06, SD; Spilia - Madari, 1250m alt., 17.12.06, MM, V. Mavrokolympos: Agios Neophytos Garrigue CY 408-3, 13.01.06, SD. Periochi Mitserou: Mitsero, Pinewood \& valley CY203-2, MM, V, AD, SD; Mitsero-Agios Panteleimonas, 11.03.06, MM, V, AD, SD. Platy: Platy area crossroad Kelefos-Kaminaria- Milikouri, 19.02.05, MG. Skoulli: Polis Camp river CY409-3, 15.01.06, SD; Goudi Oak CY409-1, 15.01.06, AD. Vouni Panagias: Makries Limnes Chasanpoulion - Eryfiou - Profitis Ilias, 23.12.04, V; Profitis Ilias, 1.02.05, MG; Vloudkia, 23.12.04, MG; Agia Moni south of Panagia, 22.12.04, MG. Xeros Potamos: Nata Pine CY407-5, 6.02.06, SD. 


\section{Comments on systematic characters}

The slug is easy to recognise even based solely on its external appearance. As emphasised by Rähle (1984), the species is characterised by very little variation of the characters which are regarded as diagnostic: the external colour pattern on the body, the penis shape, with its external and internal accessory structures, and the absence of a rectal caecum. Only the appendix at the posterior end of the penis may vary in shape. This constancy of characters is exceptional within the genus Deroceras. In all likelihood it is endemic to Cyprus. Otherwise, a slug with such a characteristic appearance would have been noticed elsewhere.

\section{Deroceras famagustensis Rähle, 1991}

It had not been reported from any NATURA 2000 site.

We found it in (Fig. 1c): Dasos Lemesou - Periochi Kyparisia: Lemesos forest, Foinikaria, 2.1.2005, MG. Dasos Machaira: Gionia Valley north (CY204-3), 17.03.06, SD; Lazania CY 204-7, 27.12.06, SD; Kapedes (CY204-1), 17.03.06, MM; Kiona - 1380 m alt., 22.12.06, MM, V; Machairas Kyprovasa-Arkatzi tou Klosmatou, 22.12.06, MM, V; Gionia Camp CY204-2, 17.03.06, SD; Philani Pine CY204-6, 27.12.06, SD. Kavo Gkreko: Paralimniou lake, 9.1.2005, MG. Madari Papoutsa: Papoutsa 1240 m alt., 20.12.06, MM; Xyliati dam, low, 21.12.06, MM, V. Maroullena: Maroulena's Pine, 23.12.06, D.

\section{Comments on systematic characters}

We found only unspotted specimens, which is in agreement with Rähle (1991), though the mantle often gives an impression of being speckled with a dark pigment. Thus the colour is not uniform. The back, outside the mantle, is covered by a pattern in the form of a dark reticulation following the system of skin grooves; the dark pigment concentrates in these grooves. Most specimens have thin and soft skin. The penis is thin-walled, of varying shape. Inside it, complicated structures adhering to the penis wall form a kind of pocket. When everted, they form a nearly circular shallow bowl or a slightly concave shield. Rähle (1991) mentions the absence of a stimulator, but apparently this structure should be regarded as one of an unusual shape. Its position within the penis, as well as when everted, clearly indicates that this is its role. The caecum is vestigial. This species is endemic to Cyprus.

There are three Natura 2000 areas, namely Drymou, Stavros tis Psokas and Kritou Marotou, where we found only juvenile Deroceras. 


\section{Family Limacidae Rafinesque, 1815}

\section{Limax flavus Linnaeus, 1758}

This species had been reported from Akrotirio Aspro - Petra tou Romiou and Ethniko Dasiko Parko Troodous (Rähle 1991).

We found it in (Fig. 2a): Dasos Lemesou - Periochi Kyparisia: Germasogeia north, at the stream (CY20), 20.2.00, V. Dasos Pafou: Kedron valley, 13.3.05, MG. Ethniko Dasiko Parko Troodous: Caledonian Falls, 22.11.05, MM. Koilada Limnati: Palia Korfi, river below at Limnatis bridge, 5.12.04, MG; Palia Korfi, approx. $500 \mathrm{~m}$ alt, Limnatis valley, 4.12.04, MM. Madari - Papoutsa: Xyliati dam, low, 21.12.06, MM.

\section{Family Milacidae Ellis, 1926}

\section{Milax barypus Bourguignat, 1866}

It had not been reported from any NATURA 2000 site.

We found it in (Fig. 2b): Kavo Gkreko: Gkreko cape at Agiol Anargyrol CY24, 21.02.00, MM; Cavo Gkreko, 9.01.2005, M. Lefkaron: Lefkara - Agios Minas, 25.02.06, MG ? juv.

This species is endemic to Cyprus.

\section{Milax riedeli Wiktor, 1986}

This species had not yet been reported from the island.

We found it in (Fig. 2c): Madari - Papoutsa: Polystypos with hazel, 17.12.06, MM, V ? juv.; Lagoudera valley, 20.12.06, MM, V, SD.

The above list represents the current state of knowledge of slug diversity within the NATURA 2000 areas of conservation of Cyprus Island. To provide a more complete picture of the fauna we have to add that Bourguignat (1853) recorded Limax antiquorum A.E. Férussac, 1819 and Limax variegatus Férussac [= Limax variegatus Draparnaud 1805] from the island, but he said that "they do not look like the true European species (naming L. cinereus)". It will be possible to clarify these names after knowing the slugs in the whole island and not only in the areas of conservation. Also Rähle (1991) listed Deroceras cyprium but this most probably refers to D. berytensis.

\section{Discussion}

In the Natura 2000 areas of conservation there are six slug species, one of them, $M$. riedeli, a new record for the whole island. According to the literature there were only 

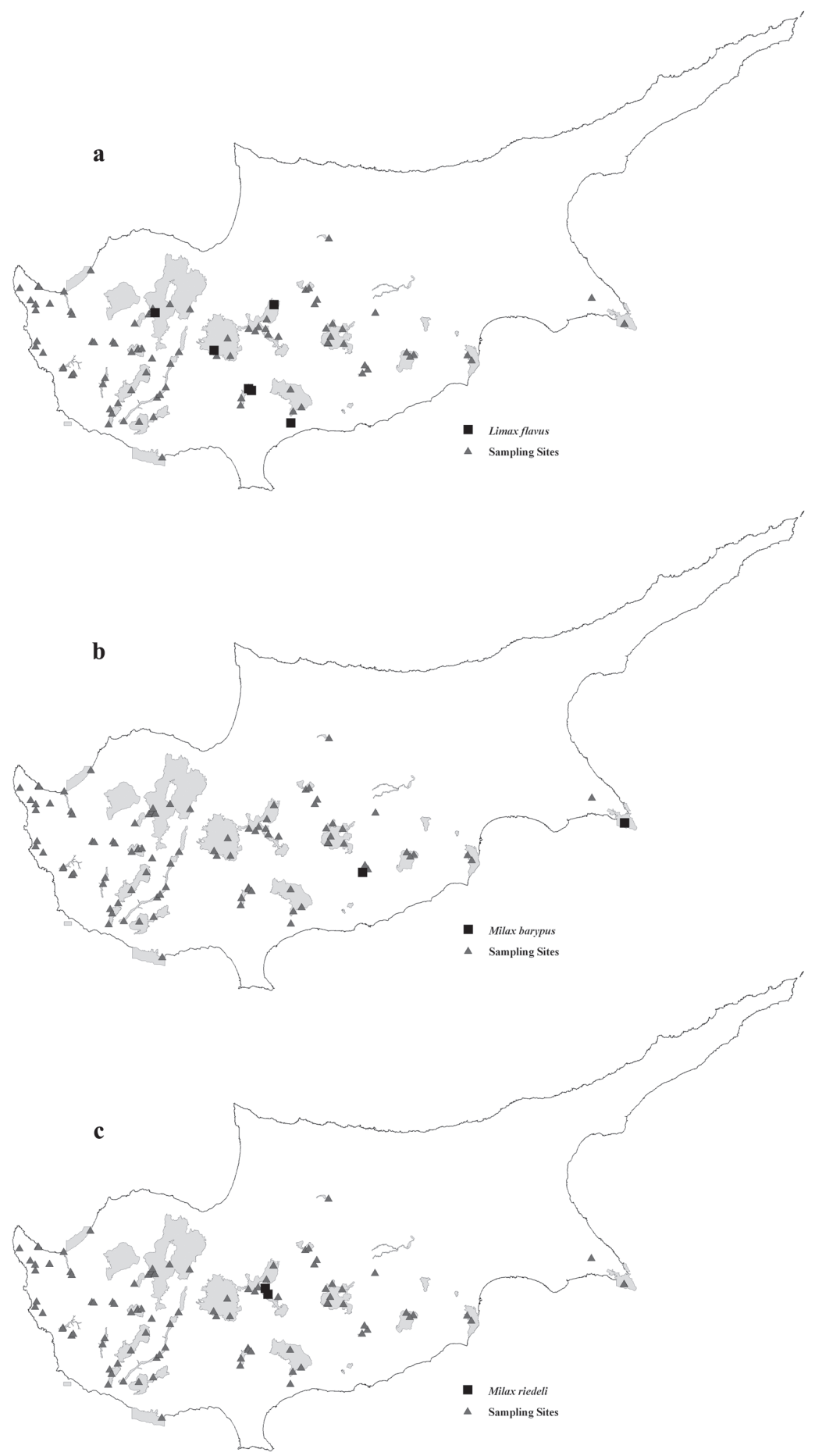

Figure 2. Distribution of a L. flavus $\mathbf{b}$ M. barypus and c M. riedeli in the Natura 2000 areas of conservation in Cyprus Island. 
three species known from the Natura 2000 areas; thus we have doubled the number of species. Tandonia sowerbyi is the only species that has been recorded from the island (Rähle 1991; pers. obs.) but still not in the NATURA 2000 areas. However, we cannot exclude the possibility of finding this species in one of the areas in the future, since in the Mediterranean it can be found in undisturbed as well as disturbed places (Wiktor 2001). The slug fauna in the NATURA 2000 areas of conservation of Cyprus appears at first glance equally rich as the corresponding areas of the island of Crete (seven species on Crete, Vardinoyannis 1994, Wiktor et al. 1994). However, on Crete there are 10 slug species on the whole island, and thus only $70 \%$ of the slug fauna is found in the conservation areas, compared to $85.7 \%$ on Cyprus. It seems that the slug fauna of Cyprus is well represented in the NATURA 2000 areas, regardless on which basis these areas were proposed.

$D$. chrysorroyatissensis and $D$. berytensis are the most widespread species, both present in 19 areas; but the latter is distributed all around the island, while $D$. chrysorryatissensis has a more restricted distribution. All the other species are found in 2-5 areas of conservation.

Three species are endemic to the island, namely D. chrysorryatissensis, D. famagustensis and $M$. barypus. One species, $M$. riedeli, is distributed on Cyprus and the southeast coast of Turkey (Schütt 2005), while L. flavus is found all around the Mediterranean and Europe (Wiktor 2001) and D.berytensis all around the eastern Mediterranean (Heller 2009; Wiktor 2000; Schütt 2005).

There are five NATURA 2000 areas where no slugs were found - Ethniko Dasiko Parko Rizoelias, Asgata, Lympion - Agia Anna, Peristerona and Koilada Kargotis. In the first two areas the substrate is mainly gypsum which is most probably the reason for their absence. In the other areas the substrate is limestone, and the vegetation is Mediterranean scrubland (maquis and phrygana). Based on all the characteristics of these areas there is no obvious explanation for the absence of slugs, and we consider it as possible that slugs might be found in the future.

The richest Natura 2000 area is Madari - Papoutsa, in the center of the island, with five of the six species present (Fig. 3). This is followed by Dasos Lemesou - Periochi Kyparisia. There is only one site, Xyliati dam, where all three Deroceras species cooccur; additionally, at this site L. flavus was also found. In most other localities there is only one slug species present, usually $D$. berytensis. Milacidae are very restricted: each species has been found in only two of the NATURA 2000 areas.

D. chrysorroyatissensis was known from very few localities in the southwest part of the island (Rähle 1991; Wiktor 2001) but with our study it appears that this species has a wider distribution, it is absent from the northern and the easternmost part of Cyprus.

D. famagustensis had been reported only from Cavo Gkreco, the southeastern peninsula of the island, but we found it in the central part of Cyprus. Further studies could enlarge its known distribution still further.

Until recently, L. flavus had been known only from sites near human settlements, but we found it also in more natural areas. 


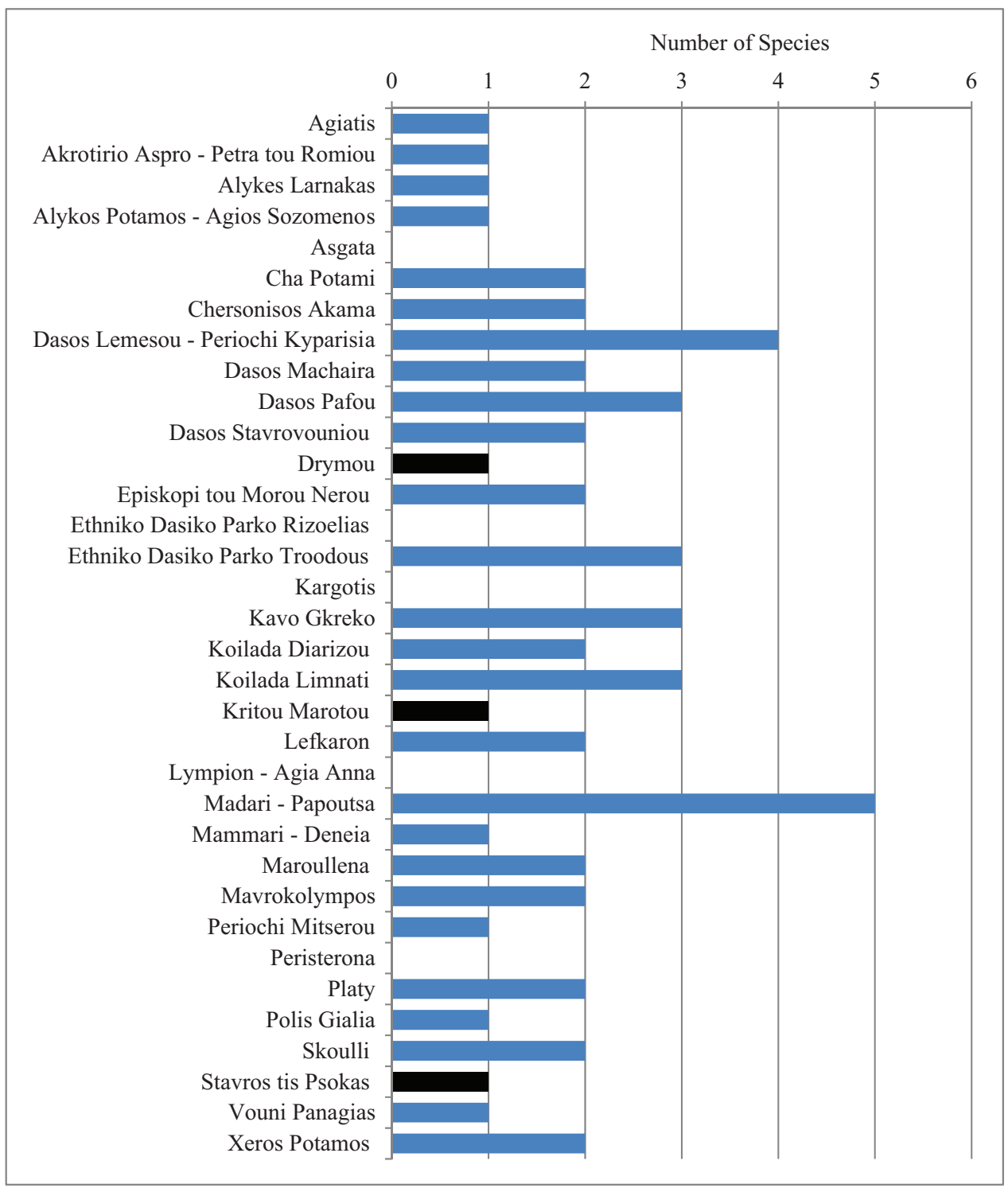

Figure 3. Number of slug species in each Natura 2000 area. Bars in black depict juvenile individuals.

$M$. barypus was known from three suburban areas in the eastern and northern part of the island but we also found it in central Cyprus.

$M$. riedeli is recorded for the first time from the island. It is distributed in the central part of the island in the area of Madari-Papoutsa.

The presence of the slug species does not seem to correlate with vegetation, rainfall, altitude or the substrate of the site they were found.

All slugs were active only during the wet period; L. flavus in the urban areas is an exception, as it was found active also during the dry season, even in summer (pers. obs.). 
In the future we will present more data on this group since we are currently studying the terrestrial malacofauna of the whole island.

\section{Acknowledgments}

We wish to thank Maria Tzatzi for her valuable help in the laboratory work, Manolis Nikolakakis for his help with the GIS presentation, and Heike Reise for reviewing the manuscript.

This work was part of the research project "The study of land snails in the NATURA 2000 areas- SUSTDEV/0104/05" funded by the Research Promotion Foundation of Cyprus.

\section{References}

Bourguignat MJR (1853) Catalogue raisonne des mollusques terrestres et fluviatiles recueillis par M.F. de Saulcy pendant son voyage en Orient. Gide \& Baudry, Paris, 96 pp, 4 pl.

Dimitrakopoulos PG, Memtsas D, Troumbis AY (2004) Questioning the effectiveness of the Natura 2000 Special Areas of Conservation strategy: the case of Crete. Global Ecology and Biogeography, 13: 199-207. doi: 10.1111/j.1466-822X.2004.00086.x

Hesse P (1926) Die Nacktschnecken der paläarktischen Region. Abhandlungen des Archiv für Molluskenkunde, 2(1): 1-151.

Heller J (2009) Land snails of the Land of Israel. Pensoft, Sofia-Moscow, 360 pp.

Rähle W (1984) Zur Kenntnis zyprischer Deroceras-Arten (Pulmonata: Agriolimacidae). Archiv für Molluskenkunde, 115: 43-50.

Rähle W (1991) Zur Kenntnis der Nacktschneckenfauna der Insel Zypern (Gastropoda, Pulmonata: Milacidae, Limacidae, Agrolimacidae). Malakologische Abhandlungen, 15: 141-148.

Schütt H (2005) Turkish Land Snails. Verlag Natur \& Wissenschaft, Solingen, 559 pp.

Simroth H (1906) Ueber einige Reihe von Nacktschnecken, die Herr Dr. Cecconi auf Cypern und in Palaestina gesammelt hat. Nachrichtsblatt der deutschen malakozoologischen Gesellschaft, 38: 17-24 and 84-91.

Wiktor A (2000) Agriolimacidae (Gastropoda: Pulmonata) - a systematic monograph. Annales Zoologici, 49(3): 347-590.

Wiktor A (2001) Fauna Graeciae. VIII. The slugs of Greece (Arionidae, Milacidae, Limacidae, Agriolimacidae-Gastropoda, Stylommatophora). Hellenic Zoologic Society \& Natural History Museum of Crete, Greece, $240 \mathrm{pp}$.

Wiktor A, Vardinoyannis K, Mylonas M (1994) Slugs of the Greek Southern Aegean Islands (Gastropoda terrestria nuda: Milacidae, Agriolimacidae et Limacidae). Malakologische Abhandlungen, 17: $1-36$.

Vardinoyannis K (1994) Biogeography of land snails in the South Aegean Island Arc (in greek). PhD Thesis, Athens, Greece: University of Athens. 